\title{
Pregnancy Outcomes in HIV-Infected Women Receiving Long-Term Isoniazid Prophylaxis for Tuberculosis and Antiretroviral Therapy
}

\author{
Allan W. Taylor, ${ }^{1}$ Barudi Mosimaneotsile, ${ }^{2}$ Unami Mathebula, ${ }^{2}$ Anikie Mathoma, ${ }^{2}$ \\ Ritah Moathlodi, ${ }^{2}$ Irene Theebetsile, ${ }^{2}$ and Taraz Samandari ${ }^{2,3}$ \\ ${ }^{1}$ Division of HIV/AIDS Prevention, Centers for Disease Control and Prevention (CDC), National Center for HIV/AIDS, Viral Hepatitis, \\ STD, and TB Prevention, 1600 Clifton Road, NE MS E-45, Atlanta, GA 30333, USA \\ ${ }^{2}$ BOTUSA, CDA Gaborone and Francistown, Plot 14818 Lebatlane Road, Phase One, Gaborone, Botswana \\ ${ }^{3}$ Division of Tuberculosis Elimination, CDC, 1600 Clifton Road NE, Mailstop E-45, Atlanta, GA 30333, USA
}

Correspondence should be addressed to Allan W. Taylor; ataylor2@cdc.gov

Received 8 November 2012; Accepted 28 January 2013

Academic Editor: Gregory T. Spear

Copyright (c) 2013 Allan W. Taylor et al. This is an open access article distributed under the Creative Commons Attribution License, which permits unrestricted use, distribution, and reproduction in any medium, provided the original work is properly cited.

\begin{abstract}
Objective. While 6- to 12-month courses of isoniazid for tuberculosis prevention are considered safe in pregnant women, the effects of longer-term isoniazid prophylaxis or isoniazid in combination with antiretroviral therapy (ART) are not established in humanimmunodeficiency-virus-(HIV-) infected women who experience pregnancy during the course of therapy. Design. Nested study of pregnancy outcomes among HIV-infected women participating in a placebo-controlled, TB-prevention trial using 36 months daily isoniazid. Pregnancy outcomes were collected by interview and record review. Results. Among 196 pregnant women, 103 (52.6\%) were exposed to isoniazid during pregnancy; all were exposed to antiretroviral drugs. Prior to pregnancy they had received a median of 341 days (range 1-1095) of isoniazid. We observed no isoniazid-associated hepatitis or other severe isoniazid-associated adverse events in the 103 women. Pregnancy outcomes were 132 term live births, 42 premature births, 11 stillbirths, 8 low birth weight, 6 spontaneous abortions, 4 neonatal deaths, and 1 congenital abnormality. In a multivariable model, neither isoniazid nor ART exposure during pregnancy was significantly associated with adverse pregnancy outcome (adjusted odds ratios $0.6,95 \%$ CI: $0.3-1.1$ and 1.8, 95\% CI 0.9-3.6, resp.). Conclusions. Long-term isoniazid prophylaxis was not associated with adverse pregnancy outcomes, such as preterm delivery, even in the context of ART exposure.
\end{abstract}

\section{Introduction}

Tuberculosis (TB) is a major cause of morbidity and mortality for HIV-infected persons [1]. A clinical trial was recently completed in Botswana, in which a 36-month course of isoniazid prophylaxis against TB (IPT) was highly efficacious in reducing the risk of TB in tuberculin-skin-test-positive adults [2] when compared with a shorter-term regimen. Based upon this and other evidence [3], the World Health Organization recommended that in countries with high TB transmission, national health programs consider providing 36-month isoniazid prophylaxis for persons living with HIV [4]. The HIV epidemic disproportionately affects women in developing countries [5]. As availability of antiretroviral medications for therapy (ART) and for prevention of mother-to-child HIV transmission (PMTCT) has been rapidly scaled up [6], and with increasing health, pregnancy is common. While 6- to 12month courses of isoniazid are considered safe in pregnancy [7], our objective was to describe the effects of longer-term isoniazid prophylaxis or simultaneous isoniazid and ART in pregnant HIV-infected women.

\section{Methods}

2.1. Study Population. A cohort of 1995 HIV-infected adults was enrolled at eight clinics located in Francistown and Gaborone, Botswana, from 2004 to 2006. Details of 
enrolment are provided elsewhere [8]. This double-blind, randomized, placebo-controlled trial gave participants 6 months of isoniazid $(300-400 \mathrm{mg} /$ day) plus vitamin B6 $(25 \mathrm{mg} /$ day $)$, after which either isoniazid or placebo $(1: 1)$ was provided for 30 months longer. Participants returned monthly for medication refills. Per national policy at the time, if a woman became pregnant and was not already receiving ART, she was referred to an antenatal clinic for free short-course zidovudine (AZT) prophylaxis from week 34 of gestation (if her CD4+ T-cell count was $>200 / \mathrm{mm}^{3}$ ) or ART if her CD4+ T-cell count was $<200 / \mathrm{mm}^{3}$. Pregnancy outcome data were collected by maternal interview and from antenatal clinic records.

2.2. Definitions. Severe hepatitis was defined as elevation of liver enzymes to $>5$ times the upper limit of normal. Severe rash was defined as generalized erythroderma, eruption, or desquamation covering $\geq 50 \%$ of body surface area. Adverse events were determined by a blinded, independent committee to be possibly, probably, definitely, or not associated with the study medication. We defined adverse pregnancy outcome (AO) as any of the following: preterm delivery (estimated gestational age (EGA) $\leq 37$ weeks at birth), low birth weight $(<2500 \mathrm{~g})$, stillbirth (delivery of an infant with no signs of life at $\geq 28$ weeks EGA), spontaneous abortion (spontaneous termination of pregnancy $<24$ weeks EGA), neonatal mortality (death of term infant within 28 days of delivery), or any noted congenital abnormality. We defined ART as therapy with $\geq 3$ antiretroviral drugs from $\geq 2$ classes provided for treatment of HIV disease. The pregnancy outcomes analysis included only the first-born infant of the first pregnancy experienced by women during the trial pregnancies completed by February 19, 2009, and only included women who registered for PMTCT.

2.3. Statistical Methods. We performed univariate, bivariate, and multivariable analysis to investigate associations between $\mathrm{AO}$, isoniazid exposure, and ART exposure in pregnancy. Continuous variables were compared using $t$-tests or nonparametric tests. We computed bivariate odds ratios to test crude associations of a priori interest. Multivariable logistic regression models were employed to explore associations of interest while controlling for potential confounders, including maternal CD4+ T-cell count nearest the last normal menstrual period (LNMP), maternal body mass index (BMI < 18.5), and age. Analyses were conducted using SAS v9.3 (SAS Institute Inc., Cary, NC, USA).

2.4. Ethical Considerations. All participants provided informed consent. The protocol was approved by Botswana and Centers for Disease Control and Prevention (CDC) ethics committees. The trial was registered at http://www .clinicaltrials.gov/, NCT00164281.

\section{Results}

Seventy-two percent of the 1995 enrollees were female; their median age was 32 years; their median $\mathrm{CD} 4+$ count was
$297 \mathrm{cells} / \mathrm{mm}^{3}$, and $47 \%$ initiated ART during the 36-month period of observation. Of the 1436 women in the study, 721 (50.2\%) were randomized to 6 months of IPT; 715 women $(49.8 \%)$ were randomized to 36 months of IPT. A total of 268 pregnancies were observed during the trial. We excluded 29 women who were still pregnant at the time the dataset was closed or who exited the trial prior to the end of pregnancy, 23 repeat pregnancies, and 20 women without PMCT regimen data, leaving a total of 196 pregnancies in the analysis dataset (98 per arm). Some participants received placebo after 6 months of IPT or stopped taking isoniazid while remaining under observation in the trial, resulting in some women not receiving isoniazid while pregnant. Of women in the 6month isoniazid arm, 20 were exposed to isoniazid during pregnancy, and 39 were exposed to ART. Of women in the 36-month isoniazid arm, 83 were exposed to isoniazid in pregnancy, and 34 were exposed to ART.

Overall, 103/196 (52.6\%) women were exposed to isoniazid during some part of pregnancy, with 102 beginning in the first trimester, and $68 \%$ of these women had continuing exposure throughout pregnancy (Table 1). None of the women receiving isoniazid had severe hepatitis or rash either during pregnancy or in the postpartum period. Among these 103 women, 94 had begun isoniazid before pregnancy; the median duration of isoniazid receipt before the last menstrual period for these women was 341 days (range 1-1095 days).

All women were exposed to antiretroviral drugs during pregnancy: 73 (37\%) received ART, and the remainder received either short-course zidovudine (121) or zidovudine/lamivudine (AZT/3TC) (2). Of 73 women receiving ART during pregnancy, the most $(64 / 73,88 \%)$ received AZT/3TC and nevirapine; 3 received lopinavir/ritonavirbased regimens; 6 received other combinations. Women taking ART had significantly lower CD4+ T-cell counts than those taking short-course prophylaxis (median CD4+ count 239 versus 452, Wilcoxon rank-sum $P<0.0001$ ). Of the 73 women receiving ART, $62 \%$ did so during all 3 trimesters. For the 47 women who began taking ART before pregnancy, the median duration of ART before pregnancy was 347 days (range 21-2221 days). Twenty-six initiated ART during pregnancy.

Two pregnant women developed TB symptoms during their pregnancies. One started anti-TB treatment 3 months after having a live birth, and the other initiated anti-TB treatment a month after her LNMP and sustained a stillbirth 7 months later. Both successfully completed standard 6-month anti-TB treatment.

Final outcomes of the 196 pregnancies were 124 (63\%) term live births; 42 (21.4\%) premature deliveries, 8 (4\%) lowbirth-weight term infants, 11 (6\%) stillbirths, 6 spontaneous abortions (3\%), 4 neonatal deaths (2\%), and 1 congenital abnormality (talipes equinovarus, Table 1).

In bivariate analysis (Table 2), ART receipt during pregnancy was associated with $\mathrm{AO}$ (unadjusted odds ratio (uOR) 2.0, $95 \%$ confidence interval (CI) 1.1-3.5), as was increasing maternal age (uOR 1.1 per year, 95\%CI 1.0-1.1). Isoniazid exposure during pregnancy was not associated with increased odds of AO. Length of exposure to INH prior to pregnancy was also not associated with increased odds of $\mathrm{AO}$ when 
TABLE 1: Selected characteristics of subjects experiencing pregnancy during the Isoniazid Preventive Therapy Trial, Botswana, 2005-2008 $(n=196)$.

\begin{tabular}{|c|c|c|}
\hline & No. & $\%$ or range \\
\hline \multicolumn{3}{|l|}{ Characteristic $(n=196)$} \\
\hline Age (median, range) & 28 & 19-39 \\
\hline CD4+ lymphocyte count nearest LNMP (median, range) in cells $/ \mathrm{mm}^{3}$ & 368 & $41-1231$ \\
\hline CD4+ lymphocyte count near LNMP $<200$ cells $/ \mathrm{mm}^{3}$ & 31 & $16 \%$ \\
\hline Maternal BMI (median, range) & 22.9 & $15.6-36.2$ \\
\hline Maternal BMI $<18.5$ near LNMP & 17 & $19 \%$ \\
\hline Pregnancy outcome date range & & Nov 262005 to Feb 192009 \\
\hline \multicolumn{3}{|l|}{ Antiretroviral regimen in Pregnancy } \\
\hline Antiretroviral therapy ( $\geq 3$ drugs from $\geq 2$ classes) & 73 & $37 \%$ \\
\hline AZT or AZT/3TC only & 123 & $63 \%$ \\
\hline \multicolumn{3}{|l|}{ Antiretroviral therapy exposure $(n=196)$} \\
\hline Number of women on ART prior to pregnancy & 47 & \\
\hline Time on ART before pregnancy (median, range) in days* & 347 & $21-2221$ \\
\hline \multicolumn{3}{|l|}{ Timing of ART exposure during pregnancy } \\
\hline First, second, and third trimesters & 45 & \\
\hline First and second trimesters only & 7 & \\
\hline Second and third trimesters only & 8 & \\
\hline Third trimester only & 8 & \\
\hline Timing unknown & 5 & \\
\hline \multicolumn{3}{|l|}{ Isoniazid exposure $(n=103)$} \\
\hline Time on isoniazid before pregnancy (median, range) in days & 341 & $1-1095$ \\
\hline \multicolumn{3}{|l|}{ Exposed to isoniazid in pregnancy } \\
\hline First trimester only & 11 & $11 \%$ \\
\hline First and second, trimesters only & 21 & $20 \%$ \\
\hline First, second, and third trimesters & 70 & $68 \%$ \\
\hline Second trimester only & 1 & $1 \%$ \\
\hline Any trimester (total) & 103 & $100 \%$ \\
\hline \multicolumn{3}{|l|}{ Pregnancy outcome $(n=196)$} \\
\hline Live birth & 124 & $63 \%$ \\
\hline Stillbirth & 11 & $6 \%$ \\
\hline Spontaneous ("inevitable") abortion & 6 & $3 \%$ \\
\hline Neonatal death & 4 & $2 \%$ \\
\hline Premature & 42 & $21 \%$ \\
\hline Term low birth weight & 8 & $4 \%$ \\
\hline Congenital abnormality & 1 & $1 \%$ \\
\hline Maternal death & 0 & $0 \%$ \\
\hline
\end{tabular}

3TC: lamivudine; ART: antiretroviral therapy; AZT: zidovudine; BMI: body mass index; IPT: isoniazid preventive therapy; LNMP: last normal menstrual period.

${ }^{*}$ Among women with ART start date prior to LNMP $(n=47)$.

analyzed either as a continuous or categorical variable (data not shown).

In a multivariable model including all variables in Table 2 , none was significantly associated with increased odds of AO except maternal age. Addition of a dichotomous variable representing maternal CD4 count $<200$ near delivery did not significantly change any model parameter, and this variable was not retained. A separate but otherwise identical model showed no association between any variable (including ART exposure) and the outcome of preterm delivery (not shown). Similarly, another separate analysis with the same covariates but with the antiretroviral drug exposure variable dichotomized as exposure prior to pregnancy or in the first trimester versus all others demonstrated no significant association with preterm delivery (not shown).

\section{Discussion}

We observed no isoniazid-associated hepatitis or other severe isoniazid-associated adverse events in 103 women receiving 
TABLE 2: Association between adverse pregnancy outcomes and isoniazid and/or antiretroviral therapy during pregnancy in HIV-infected women, Isoniazid Preventive Therapy Trial, Botswana, 2005-2008 $(n=196)$.

\begin{tabular}{|c|c|c|c|c|c|c|}
\hline Characteristic & $\begin{array}{c}\text { Adverse outcome } \\
\text { no. }(\%)\end{array}$ & $\begin{array}{c}\text { No adverse outcome } \\
\text { no. (\%) }\end{array}$ & $\mathrm{uOR}$ & $95 \% \mathrm{CI}$ & $\mathrm{aOR}$ & $95 \% \mathrm{CI}$ \\
\hline \multicolumn{7}{|l|}{ Isoniazid exposure in pregnancy } \\
\hline Yes & $32(31.1)$ & $71(68.9)$ & 0.6 & $0.3-1.1$ & 0.6 & $0.3-1.1$ \\
\hline No & $40(43.1)$ & $53(57.0)$ & 1.0 & & 1.0 & \\
\hline \multicolumn{7}{|l|}{ Antiretroviral therapy regimen in pregnancy } \\
\hline Antiretroviral therapy ( $\geq 3$ drugs from $\geq 2$ classes) & $34(46.6)$ & $39(53.4)$ & 2.0 & $1.1-3.5$ & 1.8 & $0.9-3.6$ \\
\hline Zidovudine or zidovudine/lamivudine only & $38(31.0)$ & $85(69.1)$ & 1.0 & & 1.0 & \\
\hline \multicolumn{7}{|l|}{ Maternal CD4+ lymphocyte count nearest LNMP } \\
\hline$<200$ cells $/ \mathrm{mm}^{3}$ & $13(41.9)$ & $18(58.1)$ & 1.3 & $0.6-2.8$ & 1.0 & $0.4-2.5$ \\
\hline$\geq 200$ cells $/ \mathrm{mm}^{3}$ & $59(35.8)$ & $106(64.2)$ & 1.0 & & 1.0 & \\
\hline \multicolumn{7}{|l|}{ Maternal BMI near LNMP } \\
\hline Underweight $<18.5$ & $9(52.9)$ & $8(47.1)$ & 2.0 & $0.7-5.5$ & 2.4 & $0.8-7.1$ \\
\hline Not underweight $\geq 18.5$ & $63(35.8)$ & $113(64.2)$ & 1.0 & & 1.0 & \\
\hline Maternal age (years) & - & - & 1.1 & $1.0-1.1$ & 1.1 & $1.0-1.2$ \\
\hline
\end{tabular}

BMI: body mass index; aOR: adjusted odds ratio (adjusted for all variables shown); CI: confidence interval; uOR: unadjusted odds ratio; LNMP: last normal menstrual period.

isoniazid during pregnancy and/or immediately postpartum. Generally, isoniazid hepatitis occurs in the first months of treatment, as it did in the Botswana clinical trial [2]; pregnant women in this study had received isoniazid for a median of 341 days before pregnancy. In the earlier literature there was inconclusive evidence of potentially increased risk of isoniazid-associated hepatitis and death in pregnant or postpartum women $[9,10]$. As they have a high risk of developing active TB, the CDC and American Thoracic Society recommend that IPT be initiated in HIV-infected women even during the first trimester (with routine monitoring of hepatic enzymes) [7]. Botswana policy is that, while women known to be pregnant should not initiate IPT, if they do become pregnant they should continue their course of therapy without monitoring hepatic enzymes. The overall risk of isoniazid-associated death observed in this trialoccurring in two nonpregnant women among 1995 persons who initiated isoniazid prophylaxis [2] — was no higher than the established estimated rate of hepatic death and hepatitis requiring liver transplantation of approximately $1 / 1000$ in the United States [11].

Extensive use of isoniazid during pregnancy has indicated that although it readily crosses the placental barrier, the drug is not teratogenic even when given during the first trimester $[12,13]$. We report one congenital abnormality (talipes equinovarus) in a child born to a woman who was taking isoniazid near conception. The background rate for this abnormality is unknown in Botswana; in the United States this rate has been estimated at approximately $1 / 1000$ live births [14-16]. To date a prospective registry has found no apparent increase in the frequency of this condition among infants exposed prenatally to antiretroviral medications [17].

In this study we did not observe increased odds of adverse pregnancy outcomes among women receiving ART in pregnancy compared to women receiving short-course antiretroviral regimens. Previous studies offered mixed evidence of such an association. Studies from Europe found an association between ART (especially with protease inhibitorbased ART) exposure in pregnancy and preterm delivery $[18,19]$. Similarly, a study in Botswana found increased odds of small-for-gestational-age infants among women taking ART [20]. A separate study of 71 antiretroviral drugexposed pregnancies in Botswana found no difference in rates of early pregnancy loss or stillbirths between efavirenzand nonefavirenz-exposed pregnancies; only one congenital abnormality was observed (unrelated to efavirenz exposure) [21]. Studies from the United States have not generally observed such an association [22, 23], except possibly for women starting ART before pregnancy or during the first trimester, compared to later initiation [24]. Given the known association between advanced HIV disease and preterm delivery $[25,26]$, it is possible that the borderline association between ART use and adverse birth outcome observed in the unadjusted analysis of the present study was due to confounding by maternal health status. This interpretation is supported by the disappearance of the association after controlling for maternal CD4+ count.

This study provided a unique opportunity to examine pregnancy in the context of long-term INH exposure. Chief among its limitations was the small sample size, which limited the power of the study. Also, as antiretroviral medication use was not randomized, we were unable to rule out the existence of unmeasured confounders in our analysis of antiretroviral use and $\mathrm{AO}$.

In summary, long-term prophylaxis with IPT appeared to be safe in this small secondary analysis, even when pregnancy was experienced by HIV-infected women during therapy and was not associated with adverse pregnancy outcomes. 


\section{Disclosure}

The findings and conclusions in this report are those of the authors and do not necessarily represent the official position of the Centers for Disease Control and Prevention.

\section{References}

[1] A. Reid, F. Scano, H. Getahun et al., "Towards universal access to HIV prevention, treatment, care, and support: the role of tuberculosis/HIV collaboration," The Lancet Infectious Diseases, vol. 6, no. 8, pp. 483-495, 2006.

[2] T. Samandari, T. B. Agizew, S. Nyirenda et al., "6-month versus 36-month isoniazid preventive treatment for tuberculosis in adults with HIV infection in Botswana: a randomised, doubleblind, placebo-controlled trial," The Lancet, vol. 377, no. 9777, pp. 1588-1598, 2011.

[3] N. Martinson, G. Barnes, R. Msandiwa, L. Moulton, G. Gray, J. McIntyre et al., "Novel regimens for treating latent TB in HIV-infected adults in South Africa: a randomized clinical trial," in Proceedings of the 16th Conference on Retroviruses and Opportunistic Infections, abstract \#36bLB, Montreal, Canada, 2009.

[4] World Health Organization, Guidelines for Intensified Tuberculosis Case Finding and Isoniazid Preventive Therapy for People Living with HIV in Resource Constrained Settings, World Health Organization, Geneva, Switzerland, 2011.

[5] UNAIDS, AIDS epidemic update, November 2009.

[6] B. Ojikutu, A. T. Makadzange, and T. Gaolathe, "Scaling up ART treatment capacity: lessons learned from South Africa, Zimbabwe, and Botswana," Current HIV/AIDS Reports, vol. 5, no. 2, pp. 94-98, 2008.

[7] American Thoracic Society, "Targeted tuberculin testing and treatment of latent tuberculosis infection," MMWR Recommendations and Reports, vol. 49, no. RR06, pp. 1-54, 2000.

[8] B. Mosimaneotsile, A. Mathoma, B. Chengeta et al., "Isoniazid tuberculosis preventive therapy in HIV-infected adults accessing antiretroviral therapy: a botswana experience, 2004-2006," Journal of Acquired Immune Deficiency Syndromes, vol. 54, no. 1, pp. 71-77, 2010.

[9] D. E. Snider Jr. and G. J. Caras, "Isoniazid-associated hepatitis deaths: a review of available information," The American Review of Respiratory Disease, vol. 145, no. 2, pp. 494-497, 1992.

[10] A. L. Franks, N. J. Binkin, D. E. Snider Jr., W. M. Rokaw, and S. Becker, "Isoniazid hepatitis among pregnant and postpartum Hispanic patients," Public Health Reports, vol. 104, no. 2, pp. 151$155,1989$.

[11] "Severe isoniazid-associated liver injuries among persons being treated for latent tuberculosis infection-United States, 20042008," Morbidity and Mortality Weekly Report, vol. 59, no. 8, pp. 224-229.

[12] E. A. Wilson, T. J. Thelin, and P. V. Dilts Jr., "Tuberculosis complicated by pregnancy," The American Journal of Obstetrics and Gynecology, vol. 115, no. 4, pp. 526-529, 1973.

[13] D. J. Scheinhorn and V. A. Angelillo, "Antituberculous therapy in pregnancy. Risks to the fetus," Western Journal of Medicine, vol. 127, no. 3, pp. 195-198, 1977.

[14] V. Kancherla, P. A. Romitti, K. M. Caspers, S. Puzhankara, and J. A. Morcuende, "Epidemiology of congenital idiopathic talipes equinovarus in Iowa, 1997-2005," The American Journal of Medical Genetics A, vol. 152, no. 7, pp. 1695-1700, 2010.
[15] R. N. Moorthi, S. S. Hashmi, P. Langois, M. Canfield, D. K. Waller, and J. T. Hecht, "Idiopathic talipes equinovarus (ITEV) (Clubfeet) in Texas," The American Journal of Medical Genetics, vol. 132, no. 4, pp. 376-380, 2005.

[16] C. S. Chung, R. W. Nemechek, I. J. Larsen, and G. H. Ching, "Genetic and epidemiological studies of clubfoot in Hawaii. General and medical considerations," Human Heredity, vol. 19, no. 4, pp. 321-342, 1969.

[17] Antiretroviral Pregnancy Registry Steering Committee, Antiretroviral Pregnancy Registry International Interim Report for 1 January 1989 Through 31 July 2009, Registry Coordinating Center, Wilmington, NC, USA, 2009.

[18] European Collaborative Study, "Combination antiretroviral therapy and duration of pregnancy," AIDS, vol. 14, no. 18, pp. 2913-2920, 2000.

[19] C. Thorne, D. Patel, and M. L. Newell, "Increased risk of adverse pregnancy outcomes in HIV-infected women treated with highly active antiretroviral therapy in Europe," AIDS, vol. 18, no. 17, pp. 2337-2339, 2004.

[20] N. Parekh, H. Ribaudo, S. Souda et al., "Risk factors for very preterm delivery and delivery of very-small-for-gestational-age infants among HIV-exposed and HIV-unexposed infants in Botswana," International Journal of Gynecology and Obstetrics, vol. 115, no. 1, pp. 20-25, 2011.

[21] H. Bussmann, C. W. Wester, C. N. Wester et al., "Pregnancy rates and birth outcomes among women on efavirenz-containing highly active antiretroviral therapy in Botswana," Journal of Acquired Immune Deficiency Syndromes, vol. 45, no. 3, pp. 269273, 2007.

[22] R. E. Tuomala, H. Watts, D. Li et al., "Improved obstetric outcomes and few maternal toxicities are associated with antiretroviral therapy, including highly active antiretroviral therapy during pregnancy," Journal of Acquired Immune Deficiency Syndromes, vol. 38, no. 4, pp. 449-473, 2005.

[23] R. E. Tuomala, D. E. Shapiro, L. M. Mofenson et al., "Antiretroviral therapy during pregnancy and the risk of an adverse outcome," The New England Journal of Medicine, vol. 346, no. 24, pp. 1863-1870, 2002.

[24] A. P. Kourtis, C. H. Schmid, D. J. Jamieson, and J. Lau, "Use of antiretroviral therapy in pregnant HIV-infected women and the risk of premature delivery: a meta-analysis," AIDS, vol. 21, no. 5, pp. 607-615, 2007.

[25] P. Stratton, R. E. Tuomala, R. Abboud et al., "Obstetric and newborn outcomes in a cohort of HIV-infected pregnant women: a report of the women and infants transmission study," Journal of Acquired Immune Deficiency Syndromes and Human Retrovirology, vol. 20, no. 2, pp. 179-186, 1999.

[26] M. L. Newell, D. T. Dunn, C. S. Peckham, A. E. Semprini, and G. Pardi, "Vertical transmission of HIV-1: maternal immune status and obstetric factors. The European collaborative study," AIDS, vol. 10, no. 14, pp. 1675-1681, 1996. 


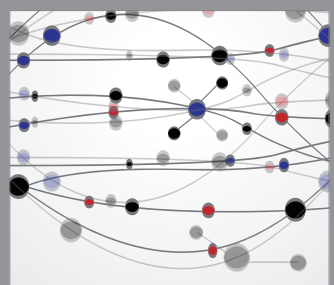

The Scientific World Journal
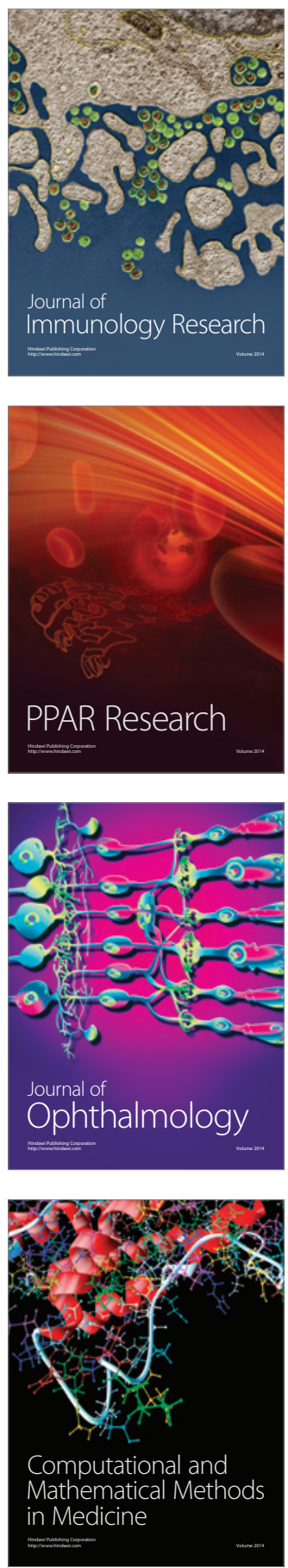

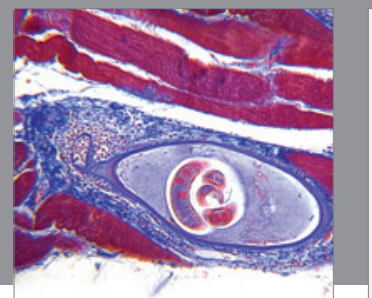

Gastroenterology

Research and Practice
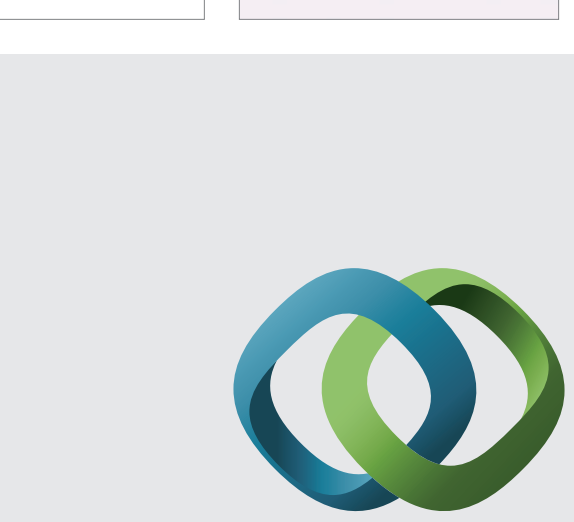

\section{Hindawi}

Submit your manuscripts at

http://www.hindawi.com
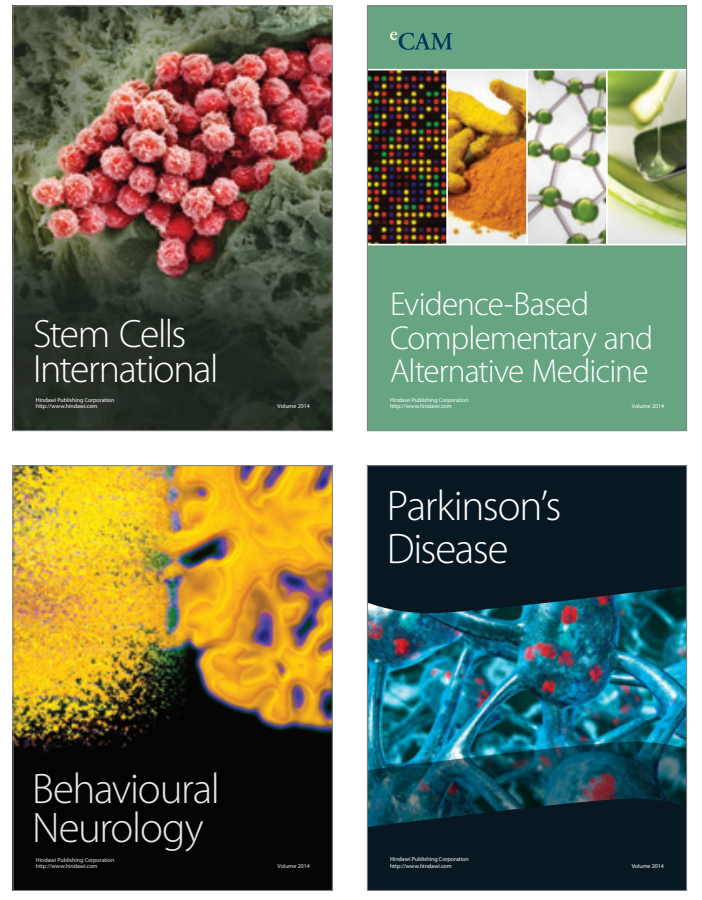
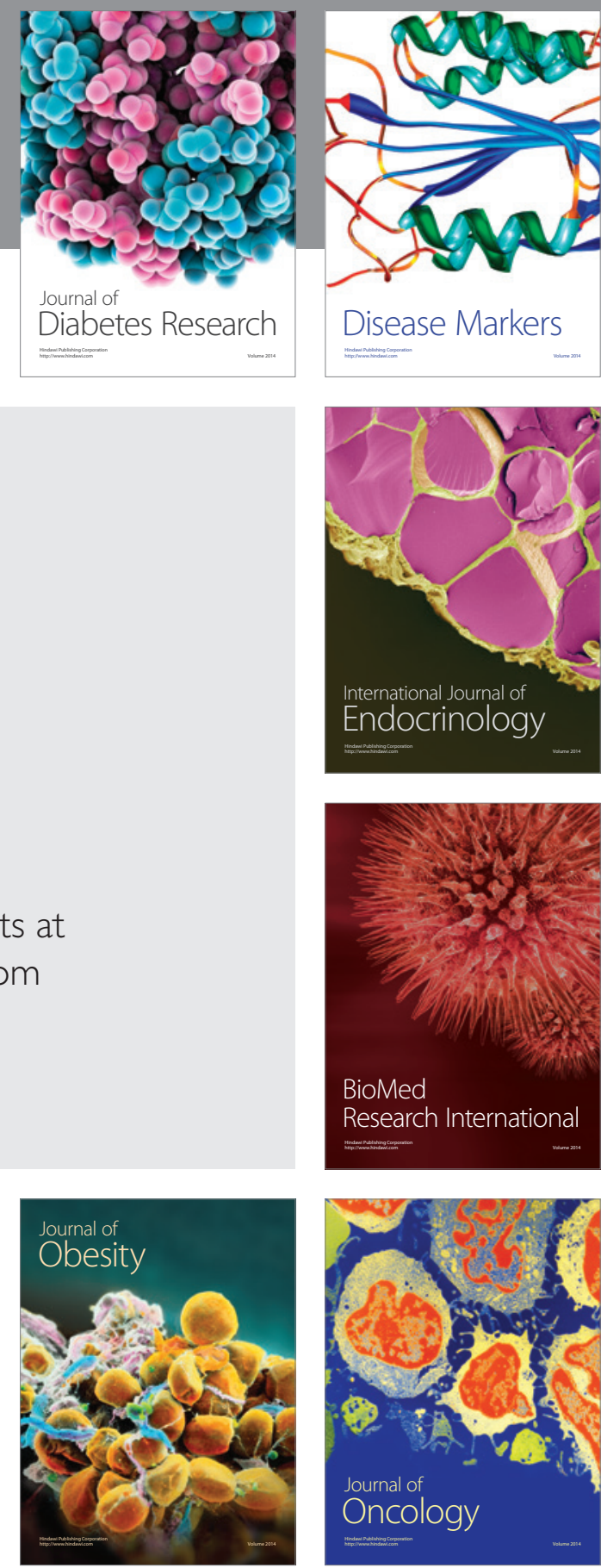

Disease Markers
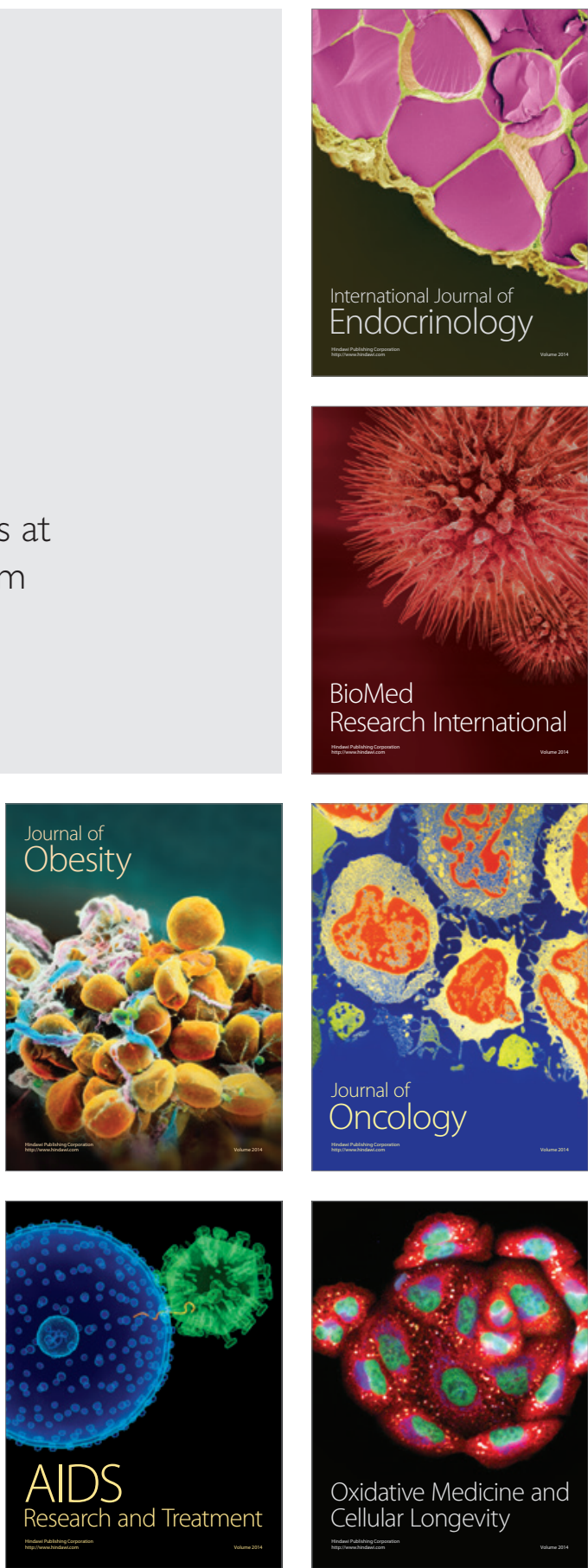